\title{
Influência dos Sintomas Pré-Procedimento na Evolução Hospitalar e Tardia das Angioplastias Carotídeas com Implante de Stent e Sistemas de Proteção Cerebral
}

\author{
Andrés Gustavo Sánchez Esteva' ${ }^{1}$ Antonio M. Kambara', Samuel M. Moreira', \\ Alaor Mendes ${ }^{1}$, Áurea J. Chaves', Manuel N. Cano', Rodolfo Staico', Luiz A. Mattos ${ }^{1}$, \\ Alexandre Abizaid ${ }^{1}$, Fausto Feres ${ }^{1}$, Amanda G. M. R. Sousa ${ }^{1}$, J. Eduardo M. R. Sousa ${ }^{1}$
}

\begin{abstract}
RESUMO
Introdução: A doença carotídea aterosclerótica (DCA) é responsável por $40 \%$ dos acidentes vasculares cerebrais (AVC). Apesar de a intervenção carotídea percutânea (ICP) ser uma alternativa à endarterectomia, ainda há poucos dados disponíveis comparando a ICP em pacientes assintomáticos (PAS) versus sintomáticos (PS). O objetivo desta análise foi avaliar essa questão em um grupo consecutivo de pacientes. Método: Foram realizadas 262 ICP em 230 pacientes consecutivos, 61 (26,5\%) PAS versus 169 (73,5\%) PS. A angiografia carotídea quantitativa (ACQ) foi realizada pré e pós-procedimento e o seguimento clínico, na fase hospitalar e aos 6 e 12 meses. Resultados: As características demográficas foram similares entre os dois grupos, sendo $31 \%$ diabéticos. Obtivemos sucesso primário em $100 \%$ dos casos, com ausência de complicações maiores em ambos os grupos. Na análise com ACQ, o diâmetro de estenose foi maior no grupo PAS $(83,4 \pm 7,6 \%$ vs. $74,9 \pm$ $12,5 \%$; $p<0,01)$, mas o grupo PS apresentou lesões mais longas $(18,3 \pm 5,7 \%$ vs. $21,7 \pm 7,4 \% ; p<0,01)$. Aos 30 dias, não houve diferença na incidência de AVC maior $(1,8$ vs. $2,0 \% ; p=0,45)$ ou menor ( 0 vs. $1,4 \% ; p=0,19)$ entre os grupos PAS e PS, respectivamente. Entre 1-12 meses, não houve casos de AVC adicionais em ambos os grupos. Constatou-se somente um caso tardio de revascularização da lesão-alvo no grupo PS e nenhuma morte de causa neurológica foi observada tanto no grupo PAS como no grupo PS. Conclusão: A ICP é uma técnica segura e eficaz, com baixa incidência de complicações aos 30 dias e 12 meses. A incidência de óbito, AVC e revascularização da lesão-alvo foi similar entre os dois grupos.
\end{abstract}

DESCRITORES: Angioplastia. Aterosclerose. Contenedores. Doenças das artérias carótidas.

\section{SUMMARY}

\author{
Influence of Symptoms Pre-Procedure in Hospital \\ and Late Evolution of Carotid Angioplasty \\ with Stent Implantation and \\ Cerebral Protection Systems
}

Background: Carotid atherosclerotic disease (CAD) is responsible for $40 \%$ of strokes. Despite percutaneous carotid intervention $(\mathrm{PCl})$ is an alternative to endarterectomy, there is little data available comparing the $\mathrm{PCl}$ in asymptomatic vs. symptomatic patients. The purpose of this analysis was to evaluate this issue in a consecutive group of cases. Methods: $262 \mathrm{PCls}$ were conducted on 230 consecutive patients, $61(26.5 \%)$ in asymptomatic vs. $169(73.5 \%)$ in symptomatic patients. Quantitative carotid angiography (QCA) was performed before and after the procedure and the clinical follow-up obtained in-hospital and at 6 and 12 months. Results: The demographic characteristics were similar between groups; $31 \%$ were diabetics. Primary success was obtained in all cases, with no major complications in both groups. QCA analysis revealed a higher diameter stenosis in asymptomatics $(83.4 \% \pm 7.6 \%$ vs. $74.9 \% \pm 12.5 \%$; $\mathrm{p}<0.01$ ), but symptomatic patients had longer lesions $(18.3 \% \pm 5.7 \%$ vs. $21.7 \% \pm 7.4 \% ; p<0.01)$. At 30 days there was no difference in the incidence of major $(1.8 \%$ vs. $2.0 \% ; p=0.45$ ) or minor strokes ( 0 vs. $1.4 \% ; p=0.19)$ for both groups. Between 1 and 12 months there have been no additional strokes. A late target vessel revascularization was observed in symptomatic patients but no neurologic deaths in both groups. Conclusion: $\mathrm{PCl}$ is safe and effective, with low incidence of complications at 1 and 12 months. The incidence of death, stroke and target vessel revascularization was similar between groups.

DESCRIPTORS: Angioplasty. Atherosclerosis. Stents. Carotid artery diseases.

\footnotetext{
1 Instituto Dante Pazzanese de Cardiologia - São Paulo, SP. Correspondência: Andrés Gustavo Sánchez Esteva. Av. Dr. Dante Pazzanese, 500 - Ibirapuera - São Paulo, SP - CEP 04012-909 E-mail: asanchez@bol.com.br

Recebido em: 7/1/2008 • Aceito em: 15/5/2008
} 
Sánchez AG, et al. Influência dos Sintomas Pré-Procedimento na Evolução Hospitalar e Tardia das Angioplastias Carotídeas com Implante de Stent e Sistemas de Proteção Cerebral. Rev Bras Cardiol Invas. 2008;16(2):211-217.

0 acidente vascular cerebral (AVC) isquêmico é a principal causa de incapacidade física e/ou mental e a terceira causa de morte na população geral. Mais de cinco milhões de pessoas morrem por ano no mundo em decorrência dessa enfermidade $^{1}$, e calcula-se que em 2020 a mortalidade por AVC será duplicada por causa do aumento da população idosa e da prevalência dos fatores de risco para aterosclerose. O AVC é a causa de cerca de $14 \%$ de todas as mortes, e a mortalidade no primeiro mês situa-se ao redor de $20 \%$. Essas repercussões do AVC (incapacidade e mortalidade) geram elevado custo social e econômico ${ }^{2}$. Em pacientes com idade maior ou igual a 65 anos, aproximadamente $80 \%$ a $85 \%$ dos AVCs são de origem isquêmica e a doença carotídea aterosclerótica (DCA) é responsável por até $50 \%$ da totalidade desses eventos ${ }^{3-10}$

Atualmente, o desenvolvimento e a melhora tecnológica dos materiais (cateteres, guias, stents, sistemas de proteção cerebral, etc.), assim como os avanços na terapêutica farmacológica, têm colocado a angioplastia carotídea como uma alternativa válida à endarterectomia para o tratamento da DCA ${ }^{11-19}$.

Entretanto, há poucos dados disponíveis a respeito das diferenças da angioplastia carotídea nos pacientes assintomáticos versus sintomáticos pré-procedimento ${ }^{20}$. O objetivo deste estudo é analisar a evolução tardia das angioplastias carotídeas, comparando os resultados (óbito de causa neurológica, AVC maior e menor, revascularização da lesão-alvo [RLA]) entre os pacientes assintomáticos versus sintomáticos.

\section{MÉTODO}

\section{Pacientes}

No período de janeiro de 2002 a outubro de 2005, foram realizadas, de forma consecutiva, 262 intervenções carotídeas percutâneas (ICP) em 230 pacientes. Foram incluídos pacientes assintomáticos e sintomáticos.

O critério para realizar angioplastia, no grupo dos pacientes assintomáticos, foi a identificação, por meio de angiografia carotídea, de estenose maior ou igual a $80 \%$ na artéria carótida interna ipsilateral; e no grupo dos pacientes sintomáticos, lesão maior ou igual a $50 \%$.

Nos pacientes sintomáticos, o procedimento foi realizado no período superior a quatro semanas após acidente isquêmico transitório (AIT) ou AVC.

Os pacientes foram divididos em dois grupos: assintomáticos (PAS, $\mathrm{n}=61$ ) e sintomáticos (PS, $\mathrm{n}=169$ ).

\section{Procedimento}

Os stents utilizados foram os disponíveis para o uso na prática clínica diária em nosso meio. O im- plante foi realizado pela técnica convencional. Foi permitida a utilização de mais de um stent por lesão ou por vaso, quando necessário.

A farmacoterapia adjunta consistiu em: 1. heparina não-fracionada (100 Ul/kg de peso), sendo administrada imediatamente antes do procedimento, com o objetivo de alcançar tempo de coagulação ativado igual ou superior a 250 segundos; 2 . ácido acetilsalicílico $(200 \mathrm{mg} / \mathrm{dia})$, iniciado 48 horas antes da intervenção e mantido indefinidamente; 3 . clopidogrel (dose de ataque de $300 \mathrm{mg}$, seguida da dose de $75 \mathrm{mg} / \mathrm{dia}$ ) ou ticlopidina (250 mg a cada 12 horas), ambos com pré-tratamento mínimo de 48 horas e mantidos durante 30 dias. Os agentes betabloqueadores, por potencializar o reflexo vasovagal durante e após a angioplastia, foram suspensos 48 horas antes e reiniciados somente 48 horas após o procedimento.

\section{Definições}

Definimos AIT como o evento neurológico que reverte o déficit dentro das primeiras 24 horas (em geral ao redor de uma hora); AVC menor, como o déficit neurológico que reverte dentro do primeiro mês; AVC maior, como o déficit persistente após o primeiro mês; e óbito de causa neurológica, quando relacionado a evento neurológico agudo. Além disso, a RLA foi definida como nova intervenção no local previamente tratado causada pela recorrência da lesão (reestenose). O sucesso clínico primário foi definido como a presença de lesão residual menor de $20 \%$ (por estimativa visual), com fluxo normal e sem novas oclusões de vasos intracerebrais, associada à ausência de eventos clínicos (morte, AVC ou cirurgia de urgência).

\section{Seguimento clínico}

Após a alta hospitalar, os pacientes foram acompanhados aos 30 dias, seis meses e um ano após o procedimento por meio de consultas ambulatoriais. A história e o exame clínico foram realizados pela equipe de intervenção endovascular e por um neurologista.

\section{Angiografia carotídea quantitativa}

As imagens angiográficas foram obtidas em múltiplas projeções ortogonais. Os pacientes foram submetidos a análise com angiografia carotídea quantitativa (ACQ) intra-stent no pré-procedimento e no pósimplante imediato (Figura 1). Todos os dados foram analisados usando o método de detecção automática de bordas. Os parâmetros quantitativos foram medidos nas fases pré-implante e imediatamente após o implante dos stents carotídeos.

\section{Análise estatística}

A comparação entre as variáveis contínuas foi realizada pelo teste $t$ de Student e expressas na forma de média e desvio padrão. As variáveis categóricas foram comparadas pela prova de qui-quadrado com 
Sánchez AG, et al. Influência dos Sintomas Pré-Procedimento na Evolução Hospitalar e Tardia das Angioplastias Carotídeas com Implante de Stent e Sistemas de Proteção Cerebral. Rev Bras Cardiol Invas. 2008;16(2):211-217.

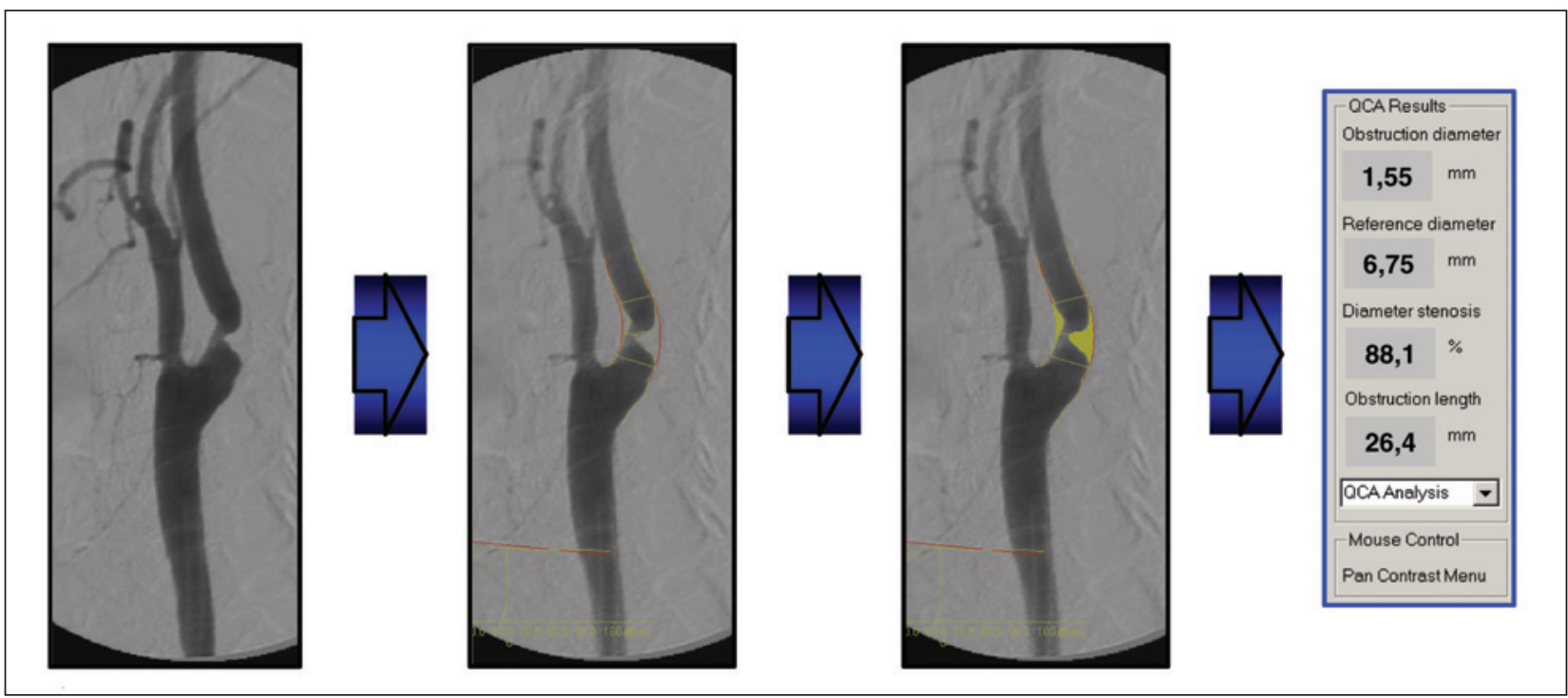

Figura 1 - Análise com angiografia carotídea quantitativa.

correção de continuidade ou pela prova exata de Fisher, quando fosse necessário, e expressas na forma de porcentagem e de freqüências absolutas e cumulativas. Valores de $\mathrm{p} \leq 0,05$ foram considerados estatisticamente significantes.

\section{RESULTADOS}

Nesta análise de 262 ICPs realizadas em 230 pacientes consecutivos, $26,5 \%$ das indicações foram em pacientes assintomáticos $(n=61)$ versus $73,5 \%$ em sintomáticos $(\mathrm{n}=169)$. As diferentes formas de apresentação clínica dos pacientes sintomáticos podem ser observadas na Tabela 1.

\section{Características demográficas}

As características demográficas basais (idade, sexo, fatores de risco para aterosclerose, infarto do miocárdio prévio, procedimentos de revascularização coronária percutâneos ou cirúrgicos prévios, lesão significante contralateral ou endarterectomia prévia ipsilateral) foram similares entre os grupos (Tabela 2). Vale destacar que $31 \%$ dos pacientes eram diabéticos.

\section{Procedimento}

As características do procedimento podem ser observadas na Tabela 3. Foram utilizados dispositivos de proteção cerebral em todos os indivíduos do grupo PAS e em 99,4\% dos indivíduos do grupo PS. Em um caso do grupo PS não foi realizada proteção cerebral, por se tratar de lesão localizada no óstio da artéria carótida comum, tratada unicamente com stent balãoexpansível.

Pré-dilatação com balão após a colocação do dispositivo de proteção cerebral foi realizada em 15\% dos pacientes, em geral nos casos de lesões muito calcificadas e/ou suboclusivas. A pós-dilatação foi realizada na maioria dos casos (96\%), com o objetivo de minimizar a lesão residual pós-implante dos stents auto-expansíveis. As freqüências de pré e pós-dilatação não diferiram entre os grupos. As pós-dilatações, de curta duração, alcançaram a pressão nominal do balão, como observado na Tabela 3. Essa abordagem foi utilizada objetivando diminuir a incidência de reações vasovagais.

O sucesso primário foi alcançado em $100 \%$ dos casos, com ausência de complicações maiores durante o procedimento nos dois grupos. Um paciente do grupo PS, portador de hipertensão arterial grave, no qual foi tratada uma lesão suboclusiva da artéria carótida interna esquerda, apresentou cifras de pressão arterial de 240/120 mmHg duas horas após o procedimento, desenvolvendo síndrome de hiperperfusão (cefaléia e confusão mental), revertida com sucesso após infusão contínua de nitroprussiato de sódio endovenoso.

\section{Características angiográficas e angiografia carotídea quantitativa}

As características angiográficas e da ACQ podem ser observadas na Tabela 4. Em 6,6\% dos pacientes do grupo PAS e em 10,1\% dos PS, foi necessária a realização de ICP bilateral estagiada, com intervalo de um mês entre o primeiro e o segundo procedimentos. Dessa forma, foram realizadas 193 intervenções no grupo PS versus 69 no grupo PAS, totalizando 262 ICP.

Não foram observadas diferenças estatisticamente significantes quando comparadas as características angiográficas (vaso tratado, características das placas, etc.) entre os dois grupos, exceto a maior freqüência 
Sánchez AG, et al. Influência dos Sintomas Pré-Procedimento na Evolução Hospitalar e Tardia das Angioplastias Carotídeas com Implante de Stent e Sistemas de Proteção Cerebral. Rev Bras Cardiol Invas. 2008;16(2):211-217.

de lesões ulceradas no grupo PS. O diâmetro de referência distal foi o medido na artéria carótida interna em $100 \%$ dos casos e o diâmetro de referência proximal, na maioria dos casos, foi igual ao diâmetro da artéria carótida comum. Na angiografia pré-procedimento, o diâmetro luminal mínimo foi menor e o porcentual de diâmetro de estenose foi maior $(83,4 \pm 7,6 \%$ vs. 74,9 $\pm 12,5 \%$; $p<0,01)$ no grupo PAS comparativamente ao grupo PS, com diâmetros de referência similares entre os grupos. O grupo PS, por outro lado, demonstrou lesões mais longas $(21,7 \pm 7,4 \mathrm{~mm}$ vs. $18,3 \pm$ $5,7 \mathrm{~mm} ; \mathrm{p}<0,01)$. A relação extensão do stent/extensão da lesão de 1,89 $\pm 0,54$ para o grupo PAS vs. $1,65 \pm 0,47$ para o grupo PS $(p<0,01)$, apesar de mostrar diferença numérica significante, indicou cobertura adequada da placa nos dois grupos.

\section{Seguimento clínico}

O seguimento clínico (Tabela 5) foi realizado em $204(88,7 \%)$ pacientes. No seguimento de 30 dias, a

TABELA 1

\begin{tabular}{lc} 
Apresentação clínica dos pacientes & sintomáticos \\
\hline & $\begin{array}{c}\text { Sintomáticos } \\
(\mathbf{n}=\mathbf{1 6 9})\end{array}$ \\
\hline AIT ocular (amaurose fugaz) & $36(21,3 \%)$ \\
AIT hemisférico (déficit motor transitório) & $49(28,9 \%)$ \\
AVC isquêmico & $78(46,2 \%)$ \\
Síncope & $6(3,6 \%)$
\end{tabular}

$\mathrm{AIT}=$ ataque isquêmico transitório; $\mathrm{AVC}=$ acidente vascular cerebral; $\mathrm{n}=$ número de pacientes. incidência tanto de AVC isquêmico maior (1,8\% vs. $2,0 \% ; p=0,45)$ como de AVC menor ( $0 \%$ vs. 1,4\%; $\mathrm{p}=0,19)$ foi similar na comparação entre os grupos. Não foram observados casos de RLA ou de morte de causa neurológica nos dois grupos nesse período. Houve apenas um caso de morte cardíaca por infarto agudo de miocárdio 18 dias após o procedimento no grupo PS.

No seguimento tardio (entre 30 dias e 12 meses), não foram observados casos de AVC isquêmico maior ou menor adicionais. Aos 10 meses de seguimento, observamos um $(0,7 \%)$ caso de RLA por reestenose intra-stent no grupo PS e nenhum caso de revascularização cirúrgica até o primeiro ano. Não houve casos de morte neurológica nesse período. A incidência de morte por outras causas foi de $1,8 \%$ para o grupo PAS versus $3,4 \%$ para o grupo PS $(p=0,27)$.

No grupo PAS, houve um caso de morte por septicemia. No grupo PS, ocorreram quatro óbitos adicionais (morte por infarto agudo de miocárdio, morte súbita, pneumonia e infecção hospitalar).

\section{DISCUSSÃO}

Na última década, foram observados avanços significativos nos procedimentos terapêuticos endovasculares. Os procedimentos percutâneos para o tratamento das artérias carótidas têm sido um dos mais beneficiados com essa evolução, tanto na terapêutica medicamentosa adjunta como na técnica e nos materiais utilizados (guias, introdutores longos, cateteres-guia, sistemas de proteção cerebral, stents auto-expansíveis de nitinol, balões, etc.). A angioplastia carotídea tornouse um método seguro, com baixa taxa de complica-

TABELA 2

Características demográficas

\begin{tabular}{lccc}
\hline & Assintomáticos $(\mathbf{n}=\mathbf{6 1})$ & Sintomáticos $(\mathbf{n}=\mathbf{1 6 9})$ & $\mathbf{p}$ \\
\hline Idade, anos & $68,8 \pm 9,6$ & $68,4 \pm 9,7$ & 0,78 \\
Sexo masculino & $43(70,5 \%)$ & $122(72,1 \%)$ & 0,40 \\
Hipertensão & $47(77,0 \%)$ & $142(84,0 \%)$ & 0,11 \\
Diabetes & $20(32,8 \%)$ & $52(30,8 \%)$ & 0,39 \\
Hiperlipidemia & $37(60,6 \%)$ & $106(62,7 \%)$ & 0,39 \\
Tabagismo & $34(55,7 \%)$ & $98(57,9 \%)$ & 0,38 \\
Creatinina $>1,5 \mathrm{mg} / \mathrm{dl}$ & $10(16,4 \%)$ & $27(15,9 \%)$ & 0,47 \\
IAM prévio & $16(26,2 \%)$ & $39(23,1 \%)$ & 0,31 \\
ATC prévia & $12(19,8 \%)$ & $38(22,5 \%)$ & 0,32 \\
CRM prévia & $18(29,5 \%)$ & $52(30,8 \%)$ & 0,43 \\
Lesão contralateral $>$ 50\% & $17(27,9 \%)$ & $54(31,9 \%)$ & 0,28 \\
Endarterectomia ipsilateral & $6(9,8 \%)$ & $10(5,9 \%)$ & 0,15 \\
\hline
\end{tabular}

IAM = infarto agudo do miocárdio; ATC = angioplastia transluminal coronária; CRM = cirurgia de revascularização do miocárdio; $\mathrm{n}=$ número de pacientes. 
TABELA 3

Características do procedimento

\begin{tabular}{|c|c|c|c|}
\hline & Assintomáticos $(n=69)$ & Sintomáticos $(n=193)$ & p \\
\hline Proteção cerebral & $69(100 \%)$ & $192(99,5 \%)$ & 0,27 \\
\hline AngioGuard $^{\circledR}$ & $49(71,0 \%)$ & $126(65,3 \%)$ & 0,19 \\
\hline FilterWire $^{\circledR}$ & $18(26,1 \%)$ & $61(31,6 \%)$ & 0,20 \\
\hline Moma $^{\circledR}$ & $2(2,9 \%)$ & $6(3,1 \%)$ & 0,47 \\
\hline Pré-dilatação & $12(17,4 \%)$ & $23(11,9 \%)$ & 0,13 \\
\hline Diâmetro do balão, mm & $3,24 \pm 0,92$ & $3,31 \pm 0,99$ & 0,61 \\
\hline Extensão do balão, mm & $19,2 \pm 6,2$ & $19,4 \pm 7,7$ & 0,84 \\
\hline Stent auto-expansível & $69(100 \%)$ & $192(99,5 \%)$ & 0,27 \\
\hline Precise Stent ${ }^{\circledR}$ & $55(79,7 \%)$ & $163(84,4 \%)$ & 0,18 \\
\hline WallStent ${ }^{\circledR}$ & $9(13,0 \%)$ & $21(10,9 \%)$ & 0,31 \\
\hline Outros & $5(7,2 \%)$ & $8(4,1 \%)$ & 0,15 \\
\hline Diâmetro do stent, mm & $7,24 \pm 0,94$ & $7,26 \pm 0,99$ & 0,82 \\
\hline Extensão do stent, mm & $31,7 \pm 7,6$ & $35,9 \pm 7,7$ & $<0,01$ \\
\hline Pós-dilatação & $65(94,2 \%)$ & $187(96,9 \%)$ & 0,16 \\
\hline Diâmetro do balão, mm & $5,48 \pm 1,29$ & $5,50 \pm 1,34$ & 0,91 \\
\hline Extensão do balão, mm & $19,1 \pm 4,0$ & $19,2 \pm 4,5$ & 0,81 \\
\hline Pressão máxima de insuflação, atm & $8,5 \pm 2,0$ & $8,9 \pm 2,2$ & 0,13 \\
\hline Tempo de insuflação, segundos & $6,69 \pm 3,28$ & $7,74 \pm 4,07$ & 0,06 \\
\hline
\end{tabular}

TABELA 4

Características angiográficas e angiografia carotídea quantitativa

\begin{tabular}{|c|c|c|c|}
\hline & Assintomáticos (n = 69) & Sintomáticos $(n=193)$ & $\mathbf{p}$ \\
\hline \multicolumn{4}{|l|}{ Intervenção carotídea } \\
\hline Unilateral & $57(82,6 \%)$ & $152(78,8 \%)$ & 0,25 \\
\hline Bilateral & $4(5,8 \%)$ & $17(8,8 \%)$ & 0,21 \\
\hline \multicolumn{4}{|l|}{ Artérias carótidas tratadas } \\
\hline Comum esquerda & $1(1,4 \%)$ & $3(1,6 \%)$ & 0,48 \\
\hline Comum direita & 0 & $1(0,5 \%)$ & 0,27 \\
\hline Interna esquerda & $35(50,7 \%)$ & $96(49,8 \%)$ & 0,44 \\
\hline Interna direita & $33(47,8 \%)$ & $93(48,2 \%)$ & 0,48 \\
\hline \multicolumn{4}{|l|}{ Características das placas } \\
\hline Calcificadas & $19(27,5 \%)$ & $45(23,3 \%)$ & 0,24 \\
\hline Irregulares & $15(21,7 \%)$ & $41(21,2 \%)$ & 0,47 \\
\hline Ulceradas & $3(4,3 \%)$ & $27(13,9 \%)$ & 0,02 \\
\hline \multicolumn{4}{|l|}{ ACQ pré-procedimento } \\
\hline Diâmetro de referência proximal, mm & $7,86 \pm 1,87$ & $7,75 \pm 1,98$ & 0,69 \\
\hline Diâmetro de referência distal, mm & $5,89 \pm 1,71$ & $5,61 \pm 1,68$ & 0,24 \\
\hline Diâmetro luminal mínimo, mm & $0,98 \pm 0,54$ & $1,41 \pm 0,69$ & $<0,01$ \\
\hline Porcentagem de estenose, $\%$ & $83,4 \pm 7,6$ & $74,9 \pm 12,5$ & $<0,01$ \\
\hline Extensão da lesão, mm & $18,3 \pm 5,7$ & $21,7 \pm 7,4$ & $<0,01$ \\
\hline Razão balão/artéria & $0,93 \pm 0,23$ & $0,98 \pm 0,29$ & 0,20 \\
\hline Razão extensão stent/lesão & $1,89 \pm 0,54$ & $1,65 \pm 0,47$ & $<0,01$ \\
\hline \multicolumn{4}{|l|}{ ACQ pós-procedimento } \\
\hline Diâmetro luminal mínimo, mm & $5,48 \pm 0,65$ & $5,29 \pm 0,48$ & 0,01 \\
\hline Porcentagem de estenose, $\%$ & $13,2 \pm 4,2$ & $12,2 \pm 4,5$ & 0,14 \\
\hline Ganho agudo, mm & $4,50 \pm 0,13$ & $3,88 \pm 0,13$ & $<0,01$ \\
\hline
\end{tabular}


Sánchez AG, et al. Influência dos Sintomas Pré-Procedimento na Evolução Hospitalar e Tardia das Angioplastias Carotídeas com Implante de Stent e Sistemas de Proteção Cerebral. Rev Bras Cardiol Invas. 2008;16(2):211-217.

TABELA 5

Seguimento clínico aos 30 dias e aos 12 meses após o procedimento

\begin{tabular}{lccc}
\hline & Assintomáticos $(\mathbf{n}=\mathbf{5 7})$ & Sintomáticos $(\mathbf{n}=\mathbf{1 4 7})$ & $\mathbf{p}$ \\
\hline 30 dias & $1(1,8 \%)$ & $3(2,0 \%)$ & \\
AVC maior ipsilateral & 0 & $2(1,4 \%)$ & 0,45 \\
AVC menor ipsilateral & 0 & 0 & 0,19 \\
RLA percutânea & 0 & 0 & - \\
Óbito de causa neurológica & 0 & $1(0,7 \%)$ & - \\
Óbito por outras causas & $1(1,8 \%)$ & $3(2,0 \%)$ & 0,27 \\
12 meses* & 0 & $2(1,4 \%)$ & 0,45 \\
AVC maior ipsilateral & 0 & $1(0,7 \%)$ & 0,19 \\
AVC menor ipsilateral & 0 & 0 & 0,27 \\
RLA percutânea & $1(1,8 \%)$ & $5(3,4 \%)$ & - \\
Óbito de causa neurológica & & 0,27 \\
Óbito por outras causas & & & \\
\hline
\end{tabular}

* Freqüências cumulativas (30 dias + 12 meses). AVC = acidente vascular cerebral; $\mathrm{n}=$ número de pacientes; RLA = revascularização da lesão-alvo.

ções, desde que realizada por operadores treinados para realizar esse tipo de procedimento. A ICP demonstrou-se equivalente à endarterectomia nos estudos randomizados CAVATAS (Carotid and Vertebral Artery Transluminal Angioplasty Study) ${ }^{11}$ e SAPPHIRE (Stenting and Angioplasty with Protection in Patients at High Risk for Endarterectomy $)^{12}$, bem como em diversos registros reunindo no total mais de 18 mil pacientes ${ }^{13-19}$.

No estudo NASCET ${ }^{10}$ (North American Symptomatic Carotid Endarterectomy Trial), foi observado que o risco de AVC no primeiro ano, nos pacientes com estenose carotídea entre $70 \%$ e $79 \%$, foi de $11 \%$, e com estenoses maiores de $90 \%$ foi de $35 \%$. No seguimento até o segundo ano dos pacientes com estenose entre $70 \%$ e $79 \%$, o risco de AVC aumentou para $26 \%$. Os pacientes com antecedente de AVC isquêmico apresentaram risco elevado de sofrer novo evento cerebrovascular da ordem de $30 \%$ a $50 \%$ em cinco anos. As diretrizes vigentes ${ }^{21-23}$ recomendam a realização de ICP nos centros com índices de eventos combinados inferiores a $3 \%$ em pacientes assintomáticos (baseado no estudo Asymptomatic Carotid Surgery ACAS $^{24}$ ) e inferiores a $6 \%$ em pacientes sintomáticos (baseado no estudo NASCET) ${ }^{10}$.

Em nossa série consecutiva de pacientes, observamos que a ICP, associada ao uso de dispositivos de proteção cerebral e implante de stent, foi uma técnica segura e eficaz nos grupos PAS e PS, com baixa incidência de eventos maiores (morte, AVC e RLA), tanto na fase hospitalar como na evolução tardia. Vale ressaltar que todos os casos de AVC após o procedimento ocorreram nos primeiros 30 dias de seguimento, não sendo observados casos de AVC entre 30 dias e um ano. A incidência de RLA percutânea foi baixa em ambos os grupos, não sendo observados casos de RLA cirúrgica. A maior porcentagem de estenose no grupo PAS pode ser explicada ao se analisar as indicações vigentes para ICP, que preconizam a utilização da técnica em casos de estenose maior ou igual a $80 \%$ em pacientes assintomáticos, e de estenose maior ou igual a $50 \%$ em pacientes sintomáticos. A maior extensão das lesões no grupo PS provavelmente decorre da maior complexidade das lesões encontradas nesses pacientes.

\section{Limitações do estudo}

Este estudo apresenta as limitações inerentes à análise retrospectiva, observacional. Estudos clínicos randomizados e controlados, com maior amostragem, são necessários para confirmar os achados deste estudo.

\section{CONCLUSÃO}

A ICP é uma técnica segura e eficaz, com baixa incidência de complicações hospitalares e a longo prazo, independentemente dos sintomas apresentados pelos pacientes antes do procedimento. A incidência de morte, AVC e RLA observada nesta série consecutiva foi similar entre os pacientes sintomáticos e assintomáticos pré-procedimento.

\section{AGRADECIMENTO}

Agradecimento ao Dr. Eduardo Missel pela colaboração na revisão detalhada do artigo.

\section{REFERÊNCIAS BIBLIOGRÁFICAS}

1. Roederer GO, Langlois $\mathrm{YE}$, Jager KA, Primozich JF, Beach KW, Phillips DJ, et al. The natural history of carotid arterial disease in asymtomatic patients with cervical bruits. Stroke. 1984;15(4):605-13. 
Sánchez AG, et al. Influência dos Sintomas Pré-Procedimento na Evolução Hospitalar e Tardia das Angioplastias Carotídeas com Implante de Stent e Sistemas de Proteção Cerebral. Rev Bras Cardiol Invas. 2008;16(2):211-217.

2. Sacco RL. Clinical practice. Extracranial carotid stenosis. N Engl J Med. 2001;345(15):1113-8

3. Hennerici M, Hülsbömer HB, Hefter H, Lammerts D, Rautenberg W. Natural history of asymptomatic extracranial arterial disease. Results of a long-term prospective study. Brain. 1987;110(pt 3):777-91.

4. Fisher CM, Gore I, Okabe N, White PD. Atherosclerosis of the carotid and vertebral arteries-extracranial and intracranial. J Neuropathol Exp Neurol. 1965;24:455-76.

5. Fields WS, Maslenikov V, Meyer JS, Hass WK, Remington RD, Macdonald M. Joint study of extracranial arterial occlusion. V. Progress report of prognosis following surgery or nonsurgical treatment for transient cerebral ischemic attacks and cervical carotid artery lesions. JAMA. 1970; 211(12):1993-2003.

6. Goldstein LB, Adams R, Becker K, Furberg CD, Gorelick PB, Hademenos G. Primary prevention of ischemic stroke: a statement for healthcare professionals from the Stroke Council of the American Heart Association. Circulation. 2001;103(1): 163-82.

7. Chambers BR, Norris JW. Outcome in patients with asymptomatic neck bruits. N Engl J Med. 1986;315(14):860-5.

8. Streifler JY, Eliasziw M, Benavente OR, Harbison JW, Hachinski VC, Barnett HJ, et al. The risk of stroke in patients with first-ever retinal vs. hemispheric transient ischemic attacks and high-grade carotid stenosis. North American Symptomatic Carotid Endarterectomy (NASCET) Trial. Arch Neurol. 1995;52(3):246-9.

9. Whisnant JP, Wiebers DO. Clinical epidemiology of transient cerebral ischemic attacks (TIA) on the anterior and posterior circulation. In: Sundt TM Jr, ed. Oclusive cerebrovascular disease: diagnosis and surgical management. Philadelphia: WB Saunders; 1987. p.60-5.

10. North American Symptomatic Carotid Endarterectomy Trial (NASCET) Collaborators. Beneficial effect of carotid endarterectomy in symptomatic patients with high-grade carotid stenosis. N Engl J Med. 1991;325:445-53.

11. Endovascular versus surgical treatment in patients with carotid stenosis in the Carotid and Vertebral Artery Transluminal Angioplasty Study (CAVATAS): a randomised trial. Lancet. 2001;357(9270):1729-37.

12. Yadav JS, Wholey MH, Kuntz RE, Fayad P, Katzen BT, Mishkel G), et al. Protected carotid-artery stenting versus endarterectomy in high-risk patients. N Engl J Med. 2004; 351(15):1493-501.

13. Roubin GS, New G, Iyer SS, Vitek JJ, Al-Mubarak N, Liu
MW, et al. Immediate and late clinical outcomes of carotid artery stenting in patients with symptomatic and asymptomatic carotid artery stenosis: a 5-year prospective analysis. Circulation. 2001;103(4):532-7

14. Mathias K. The German CAS Registry. In: $16^{\text {th }}$ ISET; Miami; 2004.

15. Wholey $\mathrm{MH}$, Wholey M, Mathias K, Roubin GS, Diethrich EB, Henry $M$, et al. Global experience in cervical carotid artery stent placement. Catheter Cardiovasc Interv. 2000; 50(2):160-7.

16. Wholey $\mathrm{MH}$, Al-Mubarek $\mathrm{N}$, Wholey $\mathrm{MH}$. Updated review of the global carotid artery stent registry. Catheter Cardiovasc Interv. 2003;60(2):259-66.

17. Albert MJ. For the publication committee for the wallstent trial of carotid vs carotid endarterectomy. Stroke. 2001;32:25

18. Bergeron P, Taube E. Comparative study of self-expanding and balloon-expanding. In: TCT Washington DC; September 11-16, 2001.

19. Theron JG, Payelle GG, Coskum O. Carotid artery stenosis: treatment with protected balloon angioplasty and stent placement. Radiology. 1996;201(3):627-36.

20. Yen MH, Lee DS, Kapadia S, Sachar R, Bhatt DL, Bajzer CT, et al. Symptomatic patients have similar outcomes compared with asymptomatic patients after carotid artery stenting with emboli protection. Am J Cardiol. 2005;95(2):297-300.

21. Bettmann MA, Katzen BT, Whisnant J, Brant-Zawadzki M Broderick JP, Furlan AJ, et al. Carotid stenting and angioplasty: a statement for healthcare professionals from the Councils on Cardiovascular Radiology, Stroke, Cardio-Thoracic and Vascular Surgery, Epidemiology, and Prevention, and Clinical Cardiology, American Heart Association. Circulation. 1998;97(1):121-3.

22. Biller J, Feinberg WM, Castaldo JE, Whittemore AD, Harbaugh RE, Dempsey RJ, et al. Guidelines for carotid endarterectomy: a statement for healthcare professionals from a special writing group of the Stroke Council, American Heart Association. Stroke. 1998;29(2):554-62.

23. ACCF/SCAI/SVMB/SIR/ASITN 2007 clinical expert consensus document on carotid stenting: a report of the American College of Cardiology Foundation Task Force on Clinical Expert Consensus Documents. J Am Coll Cardiol. 2007; 49(1):126-70.

24. Rothwell PM, Goldstein LB. Carotid endarterectomy for asymptomatic carotid stenosis. The Asymptomatic Carotid Surgery (ACAS) Trial. JAMA. 1995;273:1421-8. 